\title{
Analysis About Modules and Strings Mismatch Loss in the Photovoltaic Plant
}

\author{
Chen Min \\ Technology Support Department of GCL Energy Engineering Co, Ltd, Suzhou, China \\ Email address: \\ 563034986@qq.com \\ To cite this article: \\ Chen Min. Analysis About Modules and Strings Mismatch Loss in the Photovoltaic Plant. American Journal of Modern Energy. \\ Vol. 5, No. 3, 2019, pp. 63-68. doi: 10.11648/j.ajme.20190503.12
}

Received: May 27, 2019; Accepted: July 30, 2019; Published: August 14, 2019

\begin{abstract}
The modules mismatch loss and strings mismatch loss in PV module is theoretically and universally recognized in the PV industry [1]. But for specific projects, how to calculate these losses as well as how to reduce the impact of these losses through external rational wiring was rarely studied. In this paper, the engineering calculation method will be summed up and the relevant formulae suitable for engineering calculation or equipment configuration principles such as cables can finally be obtained by constructing the DC side equivalent circuit model of the photovoltaic power station, and also calculating of modules and strings mismatch loss data based on circuit analysis, which can in turn provide the essential theoretical basis for the accurate efficiency calculation of the photovoltaic power plant system and provide certain theoretical support for engineering drawing optimization design as well.
\end{abstract}

Keywords: Modules Loss, Strings Loss, String, Serial, Central

\section{Introduction}

The technology for the building of PV plant is developing which is becoming more necessary in power loss reduction as well as in performance ratio increase. The power loss will lead to bad incoming and power waste [2], so when the design for the PV plant is started, the way to solve these problems should be considered by every design engineers. To find this way, engineers need to analysis the value of power loss and figure out how to plan the routes of cables, how to install the facilities for reduction as well. These needs have to be solved urgently in the stage of project investment analysis, and there must be a simple, practical and accurate method for analysis and calculate loss. Based on this, the thinking of solving this problem has been aroused. Through the analysis, it is considered that the mismatch loss of photovoltaic power station in the solar time interval accounts for the vast majority of its generation time interval. In the solar time interval, the electrical characteristics of photovoltaic power station components are relatively stable, and the output of the power station is relatively idealized, which brings convenience for analysis and simplification of the model. A simple calculation model, the independent DC-side circuit model, can be established by analyzing and calculating the loss data of components in series and parallel connection in solar time. Finally, the approximate calculation formula and configuration principle can be summarized. In this way, loss data can be obtained effectively, which can provide strong theoretical support for the subsequent project, design and construction of photovoltaic power plants.

\section{Method}

\subsection{Modules Loss}

Each string of PV power plant is composed of several photovoltaic modules in series. Within each module, its I-V characteristics are different, resulting in modules losses due to different internal resistance, short circuit current and electromotive force. This section focuses on the circuit model, calculation method and avoidance principle of modules loss.

The electrical characteristics of the photovoltaic module during the start time are different from when during the stable operation time. The current keeps constant while the voltage keeps increasing when it starts. The current drops sharply but the voltage keeps unchanged while it stable operates. The circuit characteristics of the photovoltaic module are a kind of non-linear DC source [3]. The following figure is an I-W/V curve of one module, which can illustrate this characteristic: 


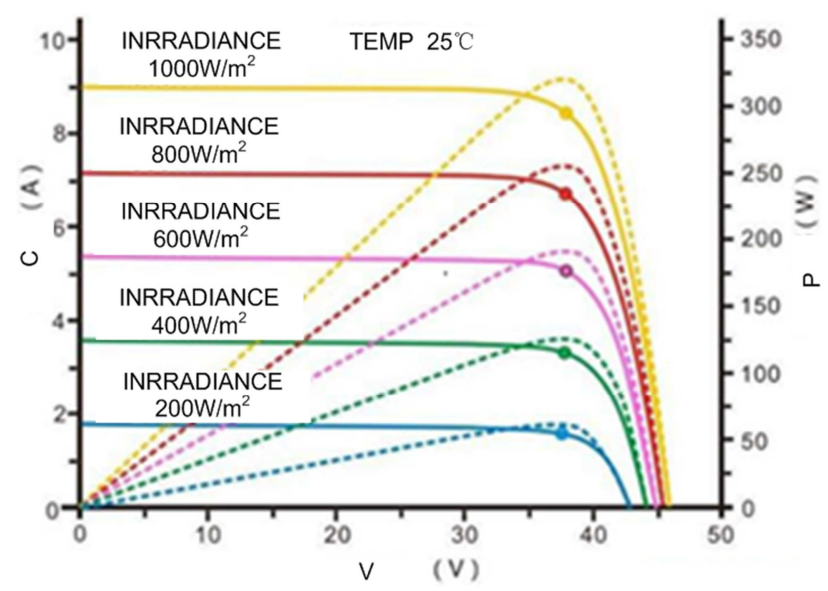

Figure 1. Module I-W/V Curve.

Although the characteristic of photovoltaic module is a non-linear source, the working characteristic of photovoltaic module is close to that of linear source in a short period of time under a specific power condition. Therefore, the equivalent model of constant voltage source can be established by using the method of linear source analysis.

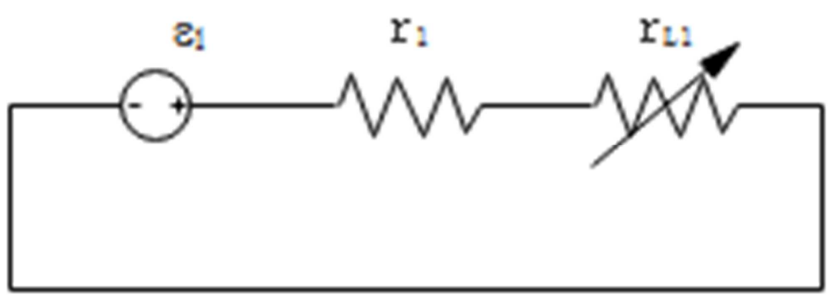

Figure 2. DC-side circuit model of module.

For a string of individual module, the maximum power should work when the load is consistent with the internal resistance [8], which can be expressed by the following formula:

$$
P_{\text {max }}=\frac{g_{1} \times \varepsilon_{1}^{2}}{4}=\frac{\varepsilon_{1}^{2}}{4 r_{1}}
$$

Since the series of photovoltaic units is composed of several modules in string, the following models can be established to simulate the series connection of multiple modules:

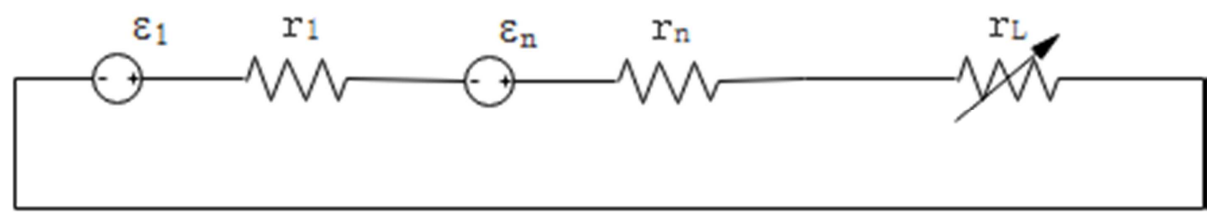

Figure 3. Multi-modules Series Circuit Model.

The maximum output power of the above string of modules shall be:

$$
P_{\max }=\frac{\left(\sum_{i=1}^{N} \varepsilon_{i}\right)^{2}}{4 \sum_{i=1}^{N} r_{i}}
$$

If each module outputs the maximum power separately, the total power is:

$$
P_{\max }=\sum_{i=1}^{N} \frac{\varepsilon_{i}^{2}}{4 r_{i}}
$$

By subtracting formula (2) from formula (3), the modules loss value can be obtained.

$$
P_{L}=\sum_{i=1}^{N} \frac{\varepsilon_{i}^{2}}{4 r_{i}}-\frac{\left(\sum_{i=1}^{N} \varepsilon_{i}\right)^{2}}{4 \sum_{i=1}^{N} r_{i}}=\frac{1}{4} \cdot \frac{\sum_{i=1}^{N-1} \sum_{j=i+1}^{N} r_{i} r_{j}\left(g_{i} \varepsilon_{i}-g_{j} \varepsilon_{j}\right)^{2}}{\sum_{i=1}^{N} r_{i}}=\frac{1}{4} \cdot \frac{\sum_{i=1}^{N-1} \sum_{j=i+1}^{N} r_{i} r_{j}\left(I_{S i}-I_{S j}\right)^{2}}{\sum_{i=1}^{N} r_{i}}
$$

Where,

$P_{L}$ is power modules loss in string,

$r_{i}$ and $r_{j}$ are including either the internal series resistance value of modules only or the total series resistance value of the internal strings resistance value plus the total series resistance value of the series connection wire. Which data to be substituted for the calculation should be specific according to the needs of the application scenario.

The formula (4) is suitable for calculating modules losses of a single string of modules consisting of a finite number of modules.

\subsection{Strings Loss}

The strings of PV power plants are assembled in parallel. Each string is involved in the combiner, its internal I-V characteristics are different and the external cable length is different. But at the combiner point, the combiner's bus will flatten all the voltages. Inverter MPPT needs to ensure maximum power output. Because of the different loop characteristics, the problem is that one of the inverters MPPT is difficult to achieve multi-channel different characteristics. For string inverters, the same MPPT usually does not exceed three strings input, so the strings loss is not obvious. For central inverters, the same MPPT will correspond to more than 20 strings, and the strings loss is more.

For a particular string, its internal characteristics are determined by the open-circuit voltage, short-circuit current, maximum power point voltage and maximum power point current of each module. The series characteristics have been analyzed in above section. The following are the calculation formula, avoidance strategy and design principle of string loss.

For a fixed time, the string is equivalent to a constant 
current source or a constant voltage source. For this kind of power supply, the circuit principle analysis shows that the external load loop needs to provide matching resistance to adapt to the internal resistance of the power supply side, so that the maximum power can be obtained. The theoretical value of matching resistance should be the corresponding internal resistance of the power supply at a certain time [4]. Based on this, the equivalent model of parallel connection of modules is established.

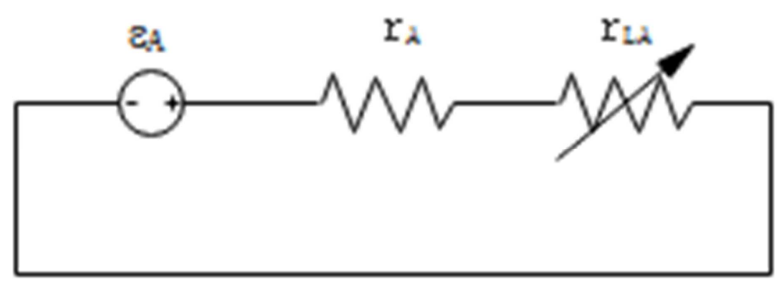

Figure 4. General circuit model after parallel connection.

Where

$\varepsilon_{\mathrm{A}}$ is always series electromotive force of photovoltaic group,

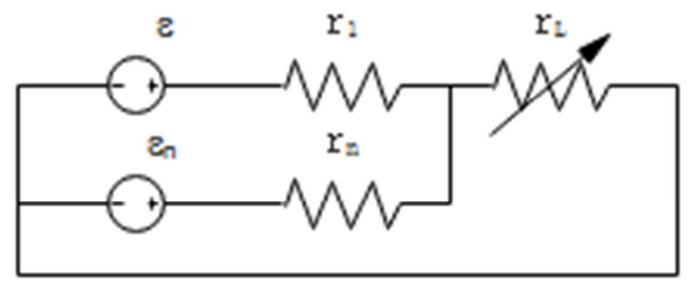

$r_{A}$ is always internal resistance plus external line loss, $\mathrm{r}_{\mathrm{LA}}$ is always matched resistance of DC side of inverter

When $\mathrm{N}$ inverters track each photovoltaic array using independent MPPT, there is theoretically a matching resistance corresponding to each array [5, 13], so the maximum power of $\mathrm{N}$ modules is:

$$
P_{\text {max }}=\frac{\sum_{i=1}^{n} g_{i} \times \varepsilon_{i}^{2}}{4}
$$

For some photovoltaic inverters, such as central inverters, one MPPT will be connected to multi-channel photovoltaic strings, so its electrical schematic will be adjusted, and the results will be quite different.

$\varepsilon_{1} \sim \mathcal{E}_{\mathrm{n}}$ or $I_{1} \sim I_{\mathrm{n}}$ is $\mathrm{N}$ strings EMF or Short Circuit Current, $r_{1} \sim r_{\mathrm{n}}$ is internal resistance value plus external line loss of each strings, $r_{\mathrm{L}}$ is DC-side matched resistance of inverters. Its resistance value varies with the change of internal resistance value of the series. Because there are many power sources and resistors in the circuit, the equivalent circuit diagram is made at first like bellowing:

Figure 5. Simplified model of parallel circuit.

According to the circuit superposition principle and the Thevenin-Norton equivalence principle [4], the final circuit diagram is equivalent to the circuit Figure 5 above, after that:

$$
\begin{gathered}
\varepsilon_{A}=\frac{\sum_{i=1}^{N} g_{i} \cdot \varepsilon_{i}}{\sum_{i=1}^{N} g_{i}} \\
g_{A}=\sum_{i=1}^{N} g_{i}=1 / r_{A}
\end{gathered}
$$

In the case of this equivalent circuit, the theoretical maximum power is:

$$
P_{\max }=\frac{\left(\sum_{i=1}^{N} g_{i} \cdot \varepsilon_{i}\right)^{2}}{4 \cdot \sum_{i=1}^{N} g_{i}}
$$

By subtracting Formula (8) from Formula (5), it can be concluded that in the case of single MPPT, the modules loss value in string is:

$$
P_{L}=\frac{1}{4} \cdot \frac{\sum_{i=1}^{N} g_{i} \times \sum_{i=1}^{N} g_{i} \times \varepsilon_{i}^{2}-\left(\sum_{i=1}^{N} g_{i} \cdot \varepsilon_{i}\right)^{2}}{\sum_{i=1}^{N} g_{i}}
$$

After the calculation is carried out, it is concluded that:

$$
P_{L}=\frac{1}{4} \cdot \frac{\sum_{i=1}^{N-1} \sum_{j=i+1}^{N} g_{i} g_{j}\left(\varepsilon_{i}-\varepsilon_{j}\right)^{2}}{\sum_{i=1}^{N} g_{i}}
$$

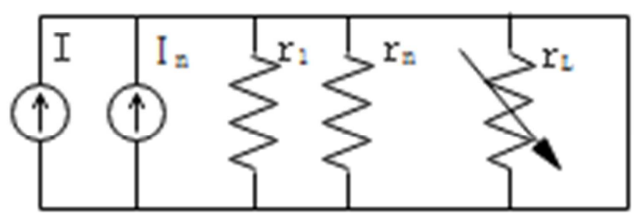

Where,

The number of molecular expansion terms should be:

$$
P_{N}^{2}=\frac{N \cdot(N-1)}{2}=\frac{N^{2}-N}{2}
$$

$g_{i}$ and $g_{j}$ are including not only the values of strings internal parallel conductance, but also the values of internal parallel conductance and the total parallel conductance of parallel wires. Which data to be substituted for calculation should be specific according to the needs of application scenarios.

\subsection{Engineering Example}

The former sections has deduced and validated the general formula of modules \& strings loss of modules. Engineering calculation can be carried out according to these formulas. This section can basically calculate the modules \& strings loss value of corresponding specifications of modules through the calculation of actual engineering cases, and find out the way and method of engineering calculation for each project in the preparatory stage of construction. 
Table 1. Electrical data measured in a string.

\begin{tabular}{|c|c|c|c|c|c|c|c|}
\hline No. & $\mathbf{P}_{\text {MAX }}$ & $\mathbf{V}_{\text {OC }}$ & $\mathbf{V}_{\text {MPP }}$ & $\mathbf{I}_{\mathrm{MPP}}$ & $\mathbf{I}_{\mathrm{SC}}$ & $\mathbf{R}_{\mathbf{I}}$ & TEMP $_{M}$ \\
\hline ACTRUAL-101 & 167.12 & 33.85 & 26.86 & 6.22 & 6.83 & 4.956076 & 45.50 \\
\hline STC-101 & 238.63 & 36.58 & 28.85 & 8.27 & 8.92 & 4.100897 & 25.00 \\
\hline 102 & 166.11 & 33.72 & 26.65 & 6.23 & 6.78 & 4.973451 & 46.70 \\
\hline 102 & 236.82 & 36.62 & 28.78 & 8.23 & 8.80 & 4.161364 & 25.00 \\
\hline 103 & 166.19 & 33.68 & 26.22 & 6.34 & 6.78 & 4.967552 & 47.00 \\
\hline 103 & 237.18 & 36.64 & 28.80 & 8.24 & 8.81 & 4.15891 & 25.00 \\
\hline 104 & 178.02 & 34.08 & 26.72 & 6.66 & 7.17 & 4.753138 & 43.60 \\
\hline 104 & 237.39 & 36.56 & 28.92 & 8.21 & 8.83 & 4.14043 & 25.00 \\
\hline 105 & 178.40 & 34.00 & 27.15 & 6.57 & 7.18 & 4.735376 & 44.30 \\
\hline 105 & 239.15 & 36.55 & 29.16 & 8.20 & 8.82 & 4.143991 & 25.00 \\
\hline 106 & 174.49 & 33.75 & 26.58 & 6.57 & 7.08 & 4.766949 & 45.20 \\
\hline 106 & 233.02 & 36.39 & 28.67 & 8.13 & 8.63 & 4.216686 & 25.00 \\
\hline 107 & 174.65 & 33.72 & 26.58 & 6.57 & 7.10 & 4.749296 & 45.10 \\
\hline 107 & 232.81 & 36.31 & 29.08 & 8.01 & 8.63 & 4.207416 & 25.00 \\
\hline 108 & 176.38 & 33.68 & 26.79 & 6.58 & 7.21 & 4.67129 & 45.30 \\
\hline 108 & 234.13 & 36.33 & 29.18 & 8.02 & 8.75 & 4.152 & 25.00 \\
\hline 109 & 176.06 & 33.65 & 26.72 & 6.59 & 7.19 & 4.680111 & 45.40 \\
\hline 109 & 235.10 & 36.26 & 28.82 & 8.16 & 8.74 & 4.148741 & 25.00 \\
\hline 110 & 191.50 & 34.15 & 27.08 & 7.07 & 7.69 & 4.440832 & 46.60 \\
\hline 110 & 241.99 & 36.82 & 29.76 & 8.13 & 8.83 & 4.169875 & 25.00 \\
\hline 111 & 191.50 & 34.15 & 27.08 & 7.07 & 7.68 & 4.446615 & 46.80 \\
\hline 111 & 242.07 & 36.84 & 29.78 & 8.13 & 8.82 & 4.176871 & 25.00 \\
\hline 112 & 189.08 & 34.09 & 26.79 & 7.06 & 7.63 & 4.46789 & 47.40 \\
\hline 112 & 238.67 & 36.83 & 29.46 & 8.10 & 8.74 & 4.213959 & 25.00 \\
\hline 113 & 189.25 & 34.09 & 27.22 & 6.95 & 7.63 & 4.46789 & 47.40 \\
\hline 113 & 238.11 & 36.82 & 29.88 & 7.97 & 8.73 & 4.21764 & 25.00 \\
\hline 114 & 186.59 & 33.73 & 26.79 & 6.96 & 7.60 & 4.438158 & 47.50 \\
\hline 114 & 234.41 & 36.43 & 29.54 & 7.94 & 8.66 & 4.206697 & 25.00 \\
\hline 115 & 186.59 & 33.75 & 26.79 & 6.96 & 7.61 & 4.434954 & 47.40 \\
\hline 115 & 234.73 & 36.43 & 29.10 & 8.07 & 8.67 & 4.201845 & 25.00 \\
\hline 116 & 187.77 & 33.82 & 26.72 & 7.03 & 7.61 & 4.444152 & 40.20 \\
\hline 116 & 226.87 & 35.68 & 28.16 & 8.06 & 8.59 & 4.153667 & 25.00 \\
\hline 117 & 187.79 & 33.80 & 26.79 & 7.01 & 7.62 & 4.435696 & 40.50 \\
\hline 117 & 228.13 & 35.81 & 28.27 & 8.07 & 8.63 & 4.149479 & 25.00 \\
\hline 118 & 191.00 & 33.88 & 26.72 & 7.15 & 7.75 & 4.371613 & 42.20 \\
\hline 118 & 234.08 & 36.07 & 28.42 & 8.24 & 8.79 & 4.103527 & 25.00 \\
\hline 119 & 191.60 & 33.86 & 26.79 & 7.15 & 7.77 & 4.357786 & 42.50 \\
\hline 119 & 234.67 & 36.03 & 28.94 & 8.11 & 8.80 & 4.094318 & 25.00 \\
\hline 120 & 190.07 & 33.72 & 26.58 & 7.15 & 7.79 & 4.328626 & 43.70 \\
\hline 120 & 233.40 & 36.01 & 28.44 & 8.21 & 8.79 & 4.096701 & 25.00 \\
\hline
\end{tabular}

The data in the table above are the electrical data of each photovoltaic module in a string measured by a large-scale ground power plant of a large photovoltaic group company [14]. The calculation is carried out according to the formula (4) in this paper like bellowing:

$P_{L}=(81.59+80.59+80.68+80.71+80.67+78.45+78.29+79.51+79.2+81.28+81.17+80.55+80.31+78.81+79+$ $76.69+77.25+79.33+79.35+79.07)-(36.58+36.62+36.64+36.56+36.55+36.39+36.31+36.33+36.26+36.82+$ $36.84+36.83+36.82+36.43+36.43+35.68+35.81+36.07+36.03+36.01)^{\wedge} 2 / 4(4.1+4.16+4.16+4.14+4.14+4.22+$ $4.21+4.15+4.15+4.17+4.18+4.21+4.22+4.21+4.2+4.15+4.15+4.1+4.09+4.1)=1592.5-728.01 \wedge 2 /(4 \times 83.21)$

$$
=0.15
$$

Under STC condition, the modules loss calculation shows that the modules loss of string consisting of 20 modules is only $0.15 \mathrm{~W}$. In this case, the modules loss of $250 \mathrm{~W}$ module is less than one thousandth of the percentage, so the modules loss of string can be neglected when selecting the same batch of modules.

Table 2. Electrical parameters of strings in a combiner area.

\begin{tabular}{|c|c|c|c|c|c|c|c|}
\hline No. & $\overline{P_{\text {MAX }}}$ & $\mathbf{V}_{\text {OC }}$ & $V_{M P P}$ & $\mathbf{I}_{\mathrm{MPP}}$ & $\overline{\mathbf{I}_{\mathrm{SC}}}$ & $\overline{\mathbf{G}_{\mathbf{I}}}$ & TEMP $_{M}$ \\
\hline ACTRUAL-1001 & 4190.52 & 742.90 & 583.80 & 7.18 & 7.85 & 0.011 & 46.40 \\
\hline STC-1001 & 5161.31 & 799.40 & 640.60 & 8.06 & 8.82 & 0.011 & 25.00 \\
\hline 1002 & 4182.62 & 742.20 & 582.70 & 7.18 & 7.86 & 0.011 & 47.20 \\
\hline 1002 & 5156.30 & 800.60 & 631.90 & 8.16 & 8.81 & 0.011 & 25.00 \\
\hline 1003 & 4180.68 & 740.60 & 581.70 & 7.19 & 7.87 & 0.011 & 48.30 \\
\hline 1003 & 5158.68 & 801.20 & 633.20 & 8.15 & 8.79 & 0.011 & 25.00 \\
\hline
\end{tabular}




\begin{tabular}{|c|c|c|c|c|c|c|c|}
\hline \begin{tabular}{|l} 
No. \\
\end{tabular} & $\mathbf{P}_{\mathrm{MAX}}$ & $V_{O C}$ & $V_{M P P}$ & $\mathbf{I}_{\mathrm{MPP}}$ & $\mathbf{I}_{\mathrm{SC}}$ & $\mathbf{G}_{\mathbf{I}}$ & $\operatorname{TEMP}_{M}$ \\
\hline 1004 & 4178.11 & 739.90 & 581.10 & 7.19 & 7.88 & 0.011 & 48.50 \\
\hline 1004 & 5158.95 & 801.20 & 633.70 & 8.14 & 8.79 & 0.011 & 25.00 \\
\hline 1005 & 4184.37 & 741.90 & 582.70 & 7.18 & 7.84 & 0.011 & 49.10 \\
\hline 1005 & 5156.41 & 804.90 & 645.60 & 7.99 & 8.73 & 0.011 & 25.00 \\
\hline 1006 & 4187.87 & 741.70 & 582.70 & 7.19 & 7.85 & 0.011 & 49.50 \\
\hline 1006 & 5141.02 & 804.90 & 646.10 & 7.96 & 8.70 & 0.011 & 25.00 \\
\hline 1007 & 4202.78 & 744.20 & 583.80 & 7.20 & 7.85 & 0.011 & 49.80 \\
\hline 1007 & 5181.52 & 808.90 & 648.50 & 7.99 & 8.72 & 0.011 & 25.00 \\
\hline 1008 & 4208.85 & 743.90 & 584.40 & 7.20 & 7.86 & 0.011 & 49.80 \\
\hline 1008 & 5178.07 & 808.10 & 648.80 & 7.98 & 8.71 & 0.011 & 25.00 \\
\hline 1009 & 4175.42 & 737.60 & 580.00 & 7.20 & 7.92 & 0.011 & 49.80 \\
\hline 1009 & 5123.59 & 801.40 & 634.50 & 8.08 & 8.76 & 0.011 & 25.00 \\
\hline 1010 & 4175.30 & 737.30 & 579.50 & 7.21 & 7.93 & 0.011 & 49.90 \\
\hline 1010 & 5127.11 & 801.10 & 634.70 & 8.08 & 8.77 & 0.011 & 25.00 \\
\hline 1011 & 4167.02 & 736.90 & 570.20 & 7.31 & 7.94 & 0.011 & 50.20 \\
\hline 1011 & 5121.10 & 801.80 & 634.90 & 8.07 & 8.77 & 0.011 & 25.00 \\
\hline 1012 & 4169.24 & 736.40 & 578.90 & 7.20 & 7.94 & 0.011 & 50.50 \\
\hline 1012 & 5118.10 & 801.40 & 635.00 & 8.06 & 8.76 & 0.011 & 25.00 \\
\hline 1013 & 4087.33 & 735.40 & 568.00 & 7.20 & 7.92 & 0.011 & 51.10 \\
\hline 1013 & 5028.66 & 802.10 & 625.30 & 8.04 & 8.72 & 0.011 & 25.00 \\
\hline 1014 & 4090.74 & 735.20 & 568.00 & 7.20 & 7.93 & 0.011 & 51.30 \\
\hline 1014 & 5036.44 & 802.50 & 625.80 & 8.05 & 8.73 & 0.011 & 25.00 \\
\hline 1015 & 4190.72 & 739.00 & 571.80 & 7.33 & 7.94 & 0.011 & 51.30 \\
\hline 1015 & 5136.12 & 805.80 & 638.90 & 8.04 & 8.72 & 0.011 & 25.00 \\
\hline 1016 & 4189.34 & 738.50 & 580.00 & 7.22 & 7.95 & 0.011 & 51.30 \\
\hline 1016 & 5135.12 & 805.30 & 638.30 & 8.05 & 8.73 & 0.011 & 25.00 \\
\hline
\end{tabular}

The above table lists 16 groups of strings data of a PV power plant. After substituting the above data with formula (9), the calculation results are as follows:

$$
\begin{gathered}
P_{L}=0.011 \times[16 \times(639040.36+640960.36+641921.44+641921.44+647864.01+647864.01+654319.21+653025.61+ \\
642241.96+641761.21+642883.24+642241.96+643364.41+644006.25+649313.64+648508.09)-(799.4+800.6+ \\
\left.801.2+801.2+804.9+804.9+808.9+808.1+801.4+801.1+801.8+801.4+802.1+802.5+805.8+805.3)^{\wedge} 2\right] \\
\div 64=0.011 \times\left[16 \times 10321237.2-(12850.6)^{\wedge} 2\right] \div 64=0.32
\end{gathered}
$$

Under STC condition, the total power of 16 strings is $82118.5 \mathrm{~W}$, and the percentage of loss in parallel is very small. Considering the field measurement of this group of data, the measured power data of 16 strings come from the terminals of strings, that is, the conductance of parallels cables is not included, and the strings loss value is understandable because of the low EMF dispersion of the modules. Generally, about $1 \%$ of the estim 0ated value is much smaller and normal, which is close to the technical index of serial inverters. At the same time, it can be concluded that the strings loss value of the combiner area mainly comes from the resistance value of the parallels cables under the actual construction conditions.

All tables and formulas are calculated in standard units above.

\section{Result}

\subsection{Several Inferences of Formula (4)}

According to formula (4), all modules losses can be calculated accurately. It is very practical for engineering calculation. Its guiding significance is self-evident. From the formula structure, the following inferences can be derived:

Inferences 1: In theory, when the short circuit current of each photovoltaic module is the same, there is no modules loss. At this time, regardless of the EMF and internal resistance of the module, it will only affect other types of losses without modules loss.

Inferences 2: The smaller the dispersion of short-circuit current, the smaller the modules loss. It can also be expressed as the more concentrated the short-circuit current is, the smaller the modules loss is.

Inferences 3: When the short circuit current difference between the two modules is too large, the modules loss can be reduced by choosing two modules with smaller internal resistance instead of two modules with too large electromotive force.

\subsection{Several Inferences of Formula (10)}

Several effective principles can be concluded from the structure of Formula (10). At the same time, all strings losses can be calculated accurately according to this formula, which conforms to the practical principle of engineering calculation. Several qualitative inferences can be summarized, and quantitative analysis will be introduced in the next section.

Inferences 1: In theory, when the strings EMF of each photovoltaic unit is the same, there is no strings loss. At this time, no matter how the external wiring is, it will only affect the other loss without strings loss.

Inferences 2: The smaller the EMF dispersion of each 
photovoltaic string is, the smaller the loss of strings is. It can also be expressed that the more concentrated the EMF of strings is, the smaller the loss of strings is.

Inferences 3: When the difference of EMF between two groups is too large, the strings loss can be reduced by concentrating the wiring length of DC cables in each string.

\section{Discussion}

In Most Articles for this question, the way to discuss modules loss or strings loss was complex and not suitable for engineering calculations, such us in this one, which abstract is as follows, Variations in the I-V characteristics of photovoltaic modules can lead to significant power loss. This study deals with an analysis of series-string losses. The current parameter of a set of PV modules is considered as a random variable following the Weibull distribution. An analytical expression has been obtained to predict these losses. A preliminary experiment that shown a good agreement with the proposed model is also presented. [16] While in this paper, every formula is simple and practical, therefore it can be used to calculate the losses before the investment for a new PV solar plant.

\section{Conclusion}

In this paper, the circuit principle of the modules \& strings loss of modules is modeled, the calculation formula is deduced, and the engineering case is calculated. Finally, it is concluded that the modules loss accounts for a very small proportion of the total mismatch losses, and the calculation can be neglected. For the strings loss, the formula (9) can be used to calculate which quickly, and the actual parameters of the modules can be obtained from the batch test report provided by the factory of the modules. According to the combination simulation, the suitable group of strings can be obtained. After one calculation being finished, the approximate range of the modules loss of the same specification module can be basically determined, so that provides a fast and accurate way for the theoretical calculation in the design stage.

For engineering construction, when conditions are permitted, priority should be given to ways to reduce the strings loss, such as reducing the open-circuit voltage dispersion rate of each string to avoid large proportion of parallel mismatch, reducing mismatch by adjusting the length of each strings cables to reduce the appropriate dispersion rate of cable length. Hoping it can also play an active role in later engineering applications or computer aided design.

\section{References}

[1] Evans R, Boreland M, Green M A. A holistic review of mismatch loss: From manufacturing decision making to losses in fielded arrays [J]. Solar Energy Materials \& Solar Cells, 2018, 174: 214-224.

[2] Han C, Lee H. Investigation and Modeling of Long-term Mismatch Loss of Photovoltaic Array [J]. Renewable Energy, $2018,121$.

[3] Li Shanshou, Zhang Xing. Modified Mathematical Modeling Method for Photovoltaic Module Engineering [J]. Electric Power Automation Equipment 2015.

[4] "Circuit Principles" 2nd edition, edited by Zhou Tingyang and Jiang Weicheng, Zhejiang University Press, 1997.

[5] Function, principle and algorithm of photovoltaic inverters MPPT. Solar photovoltaic network.

[6] Wang Yuejiao, Shenyang Normal University. The steps and methods of analyzing circuit schematic diagram.

[7] Muhyaddin J. H. Rawa, David W. P. Thomas, Mark Sumner. A Review on Recent Development of Photovoltaics and Wind Turbines.

[8] Zhiguo Zhu, Guowei Liu. MPPT Control Method for Photovoltaic System Based on Particle Swarm Optimization and Bacterial Foraging Algorithm.

[9] Samuel Sami, Jorge Rivera. A Predictive Numerical Model for Analyzing Performance of Solar Photovoltaic, Geothermal Hybrid System for Electricity Generation and District Heating.

[10] Paiguy Armand Ngouateu Wouagfack, Ariane Laurelle Ngankou, Noël Djongyang, Réné Tchinda. Electrical and Exergy Analysis of a Simple Pass Photovoltaic-Thermal (PV/T) Air Heater with Slats Under Weather Conditions of the Far Nord Region, Cameroon.

[11] Energy Loss in Solar Photovoltaic Systems Under Snowy Conditions. Energy Loss in Solar Photovoltaic Systems Under Snowy Conditions.

[12] Nuru Safarov, Sevinc Orucova, Gurban Ahmadov, Etibar Rzazadeh, Samira Cafarova. Efficiency in Solar Photovoltaic Systems Under High Temperature Conditions.

[13] Ye qiuxiang, Zheng jianli. Research and Exploitation of Photovaltaic Maximum Power Point Tracking System.

[14] Ramos, Gerber N. Field Testing Method for Photovaltaic Modules.

[15] Sun Yunlin, Chen Rongrong, Xu Shutong, Wang Xiaoyang. 30 MWp Photovaltaic Generation Demonstration Project in Fuhua Machinery City.

[16] R, Zilles, E, Lorenzo, an Analytical Model for Mismatch Losses in PV Arrays. 